\title{
Unliganded thyroid hormone receptor alpha1 impairs adult hippocampal neurogenesis
}

\author{
Richa Kapoor ${ }^{1,}$, Max van Hogerlinden ${ }^{2,}$, , Karin Wallis ${ }^{2}$, Himanish Ghosh ${ }^{1}$, Kristina \\ Nordstrom $^{2}$, Bjorn Vennstrom ${ }^{2, \#}$, and Vidita A. Vaidya ${ }^{1, \#}$ \\ ${ }^{1}$ Department of Biological Sciences, Tata Institute of Fundamental Research, Mumbai 400005, \\ India \\ ${ }^{2}$ Department of Cell and Molecular Biology, Karolinska Institutet, SE-171 77 Stockholm, Sweden.
}

\section{Abstract}

Thyroid hormone regulates adult hippocampal neurogenesis, a process involved in key functions such as learning, memory and mood regulation. We addressed the role of thyroid hormone receptor TRa1 in adult hippocampal neurogenesis, using mice harboring a TRa1 null allele (TRa1-/-), overexpressing TRa1 6-fold (TRa2-/-), and a mutant TRa1 (TRa1+/m) with a 10fold lower affinity to the ligand. While hippocampal progenitor proliferation was unaltered, TRa1-/- mice exhibited a significant increase in doublecortin-positive immature neurons and increased survival of bromo-deoxyuridine (BrdU)-positive progenitors as compared to wild-type controls. In contrast, the TRa $1+/ \mathrm{m}$ and the TRa2-/- mice, where the overexpressed TRa 1 acts as an aporeceptor, showed a significant decline in surviving BrdU-positive progenitors. TRa1-/- and TRa2-/- mice showed opposing effects on neurogenic markers like polysialylated neural cell adhesion molecule and stathmin. The decreased progenitor survival in the TRa2-l- and TRa1+/m mice could be rescued by thyroid hormone treatment, as was the decline in neuronal differentiation seen in the TRa1+/m mice. These mice also exhibited a decrease in NeuroDpositive cell numbers in the dentate gyrus, suggesting an effect on early postmitotic progenitors. Our results provide the first evidence of a role for unliganded TRa 1 in modulating the deleterious effects of hypothyroidism on adult hippocampal neurogenesis.

\section{Keywords}

hippocampus; neural stem cell; hypothyroidism; neuronal progenitor

\section{Introduction}

Thyroid hormone perturbations in development cause major neuroanatomical and neurological deficits (1). Though adult-onset hypothyroidism does not manifest as severely as developmental hypothyroidism (2), it can precipitate depressive behavior and deteriorate

Address Correspondence to: Dr. Vidita A. Vaidya Department of Biological Sciences, Tata Institute of Fundamental Research Homi Bhabha Road, Mumbai 400005 India vvaidya@tifr.res.in Or Dr. Bjorn Vennstrom Department of Cell and Molecular Biology,

Karolinska Institutet, SE-171 77 Stockholm, Sweden bjorn.vennstrom@ki.se.

Equal contributing first authors.

\#Equal contributing senior authors. 
cognitive function $(3,4)$. In particular, adult hypothyroidism impairs hippocampaldependent behaviors, resulting in learning, memory and mood related deficits $(5,6)$. Adult hippocampal neurogenesis plays an important role in these hippocampal-dependent tasks ( 7 , 8 ), and is regulated by thyroid hormone $(9,10,11)$. Decreased hippocampal neurogenesis has been postulated to contribute to the deficits in hippocampal functions observed in adultonset hypothyroidism.

Adult neurogenesis encompasses progenitor proliferation, survival and differentiation, and the maturation and functional integration of newborn neurons (12). The developmental stages of adult neurogenesis are characterized by stage-specific markers, such as nestin, NeuroD, doublecortin (DCX), polysialylated neural cell adhesion molecule (PSA-NCAM), stathmin and calretinin $(13,14)$. Adult hypothyroidism decreases progenitor survival, DCXpositive immature neuron number and neuronal differentiation, with no effect on progenitor proliferation $(9,10)$. In vitro evidence suggests a direct effect of thyroid hormone on adult hippocampal progenitors (10). However, the role of thyroid hormone receptors (TRs) and their contribution to the damaging effects of hypothyroidism on adult hippocampal neurogenesis is unknown.

TRs are transcription factors that bind thyroid hormone response elements and activate or repress target genes as ligand-receptor complexes or aporeceptors (15). TR alpha and beta genes generate several TR isoforms, of which TRa1, TRa2, TR $\beta 1$ and TR $\beta 2$ are predominant in the adult mammalian brain (2). TRa1 contributes $70-80 \%$ of total TR expression in the brain (16). TRa2 does not bind thyroid hormone, though some reports implicate TRa2 in the transcriptional repression of thyroid hormone responsive genes (17). It is of interest to note that the phenotype in TRa2-/- mice, which as a consequence of ablation of TRa2 inevitably overexpress TRa1 several-fold, has been ascribed to TRa1 aporeceptor effects in many tissues (18).

It remains unclear if the deleterious effects of hypothyroidism are due to insufficient target gene activation or a consequence of the aporeceptor acting as a transcriptional regulator (15). The focus of the present study was to investigate the role of TRa 1 in adult hippocampal neurogenesis, using TRa1-/-, TRa2-/- and TRa1+/m heterozygous mice carrying a point mutation (TRa1R384C) that lowers thyroid hormone affinity 10-fold (18, 19, 20). Further, TRa1-GFP expressing mice were utilized to address the stage-specific expression of TRa1 in adult hippocampal progenitors. Our results demonstrate a key role for TRa1 in the regulation of the postmitotic survival of adult hippocampal progenitors, and indicate that an unliganded TRa1, acting as an aporeceptor, is responsible for the deleterious effects of hypothyroidism on adult hippocampal neurogenesis.

\section{Materials and Methods}

\section{Thyroid hormone receptor mutant mice}

TRa1-/- and TRa2-/- mice were generated as previously described $(18,19)$. The mouse strain carrying the dominant negative $\mathrm{R} 384 \mathrm{C}$ mutation in $\mathrm{TRa} 1(\mathrm{TRa} 1+/ \mathrm{m})$ was generated as described previously (20). TRa1-GFP knock-in mice were constructed by inserting the coding sequence of eGFP in frame $3^{\prime}$ to exon 9 of the TRa 1 gene (Wallis et al., 2010, in 
press). Heterozygote offspring were bred against $\mathrm{C} 57 \mathrm{Bl} / 6$ for three generations and then intercrossed to generate TRa1-GFP mice homozygous for the chimeric gene. TRa1-GFP knock-in mice have normal body and organ weights, T3, T4 and TSH levels, and show no overt morphological or physiological phenotype. The Littermate mutant and wild type mice were kept at $21^{\circ} \mathrm{C}$ on a $12 \mathrm{hr}$ light/ dark cycle and two month old male mice were used in the study. Animal care procedures were in accordance with the guidelines set by the European Community Council Directives (86/609/EEC) and were approved by the Karolinska Institutet Animal Ethics committee and the TIFR Institutional Animal Ethics committee.

\section{BrdU labeling paradigms and drug treatments}

To determine if TRa1 is expressed by proliferating adult hippocampal progenitors, we injected heterozygote TRa1-GFP mice with a single intraperitoneal (i.p.) injection of the mitotic marker 5-bromo-2'-deoxyuridine (BrdU, $150 \mathrm{mg} / \mathrm{kg}$ body weight; Sigma, USA), 2 hours prior to sacrifice $(n=3)$. Male wild type mice were used as a negative control to ensure specificity of the GFP signal. To address the effects on adult hippocampal progenitor proliferation, TRa1-/- and TRa2 -/- mice as well as littermate wild type controls received a single i.p. injection of $\mathrm{BrdU}$ (100 mg/kg body weight; Sigma) and were sacrificed 2 hours later ( $n=4-5 /$ group). To address the role of an unliganded TRa 1 receptor on adult hippocampal progenitor proliferation, TRa $1+/ \mathrm{m}$ and wild type littermate controls received a single BrdU (100 mg/kg body weight) injection and were sacrificed 2 hours later ( $\mathrm{n}=4-5 /$ group).

To examine effects on the survival and differentiation of adult hippocampal progenitors, TRa1-/- and TRa2 -/- mice as well as littermate wild type controls were administered $\mathrm{BrdU}$ ( $150 \mathrm{mg} / \mathrm{kg}$ body weight) once daily by i.p. injection for 3 consecutive days and were sacrificed 28 days after the last injection ( $n=4-5 /$ group). To examine the role of an unliganded TRa1 receptor on adult hippocampal progenitor survival and differentiation, $\mathrm{TRa} 1+/ \mathrm{m}$ and wild type littermate controls received a single daily $\mathrm{BrdU}(100 \mathrm{mg} / \mathrm{kg}$ body weight) injection for three consecutive days and were sacrificed 30 days later $(n=4-5 /$ group).

Experiments to address the influence of thyroid hormone treatment on adult hippocampal progenitor survival in TRa2 -/- mice had four experimental groups: Wild type + vehicle, wild type + T3, TRa2 -/- + vehicle, TRa2-/- + T3 ( $\mathrm{n}=4-5 /$ group). The groups receiving thyroid hormone treatment were given T3 $(0.5 \mu \mathrm{g} / \mathrm{ml}$, Sigma, USA) in drinking water, as described previously (21) for 16 days prior to sacrifice.

In experiments to examine the effect of thyroid hormone treatment on adult hippocampal progenitor survival and differentiation in TRa $1+/ \mathrm{m}$ mice, there were 4 experimental groups: Wild type + vehicle, wild type + T3, TRa $1+/ m+$ vehicle, TRa $1+/ m+T 3$ ( $=4-5 /$ group). The treatment groups (wild type $+\mathrm{T} 3$, TRa $1+/ \mathrm{m}+\mathrm{T} 3$ ) received T3 $(0.5 \mu \mathrm{g} / \mathrm{ml}$ ) in drinking water as described previously (21) for 12 days. From day 10 to day 12, all animals were administered a single injection of BrdU ( $150 \mathrm{mg} / \mathrm{kg}$ body weight), and were sacrificed 30 days after the last BrdU injection. 


\section{Immunohistochemistry}

Mice were sacrificed by transcardial perfusion with 4\% PFA, brains were removed, postfixed and subsequently cryoprotected in $30 \%$ sucrose-PFA. Serial coronal sections $(30 \mu \mathrm{m})$ through the rostro-caudal extent of the hippocampus were generated using a freezing microtome (Leica, Germany). Free-floating sections were processed for BrdU immunohistochemistry as described previously (10). In brief, post DNA denaturation and acid hydrolysis, sections were incubated overnight with mouse anti-BrdU antibody (1:500, Boehringer Mannheim, USA). Sections were then exposed to secondary antibody (biotinylated anti-mouse IgG, 1:500, Vector Laboratories, USA). An avidin-biotin complex (Vector Laboratories) was used for signal amplification, which was detected using the substrate diaminobenzidine (Sigma).

For immunohistochemistry and immunofluorescence experiments to examine the expression of endogenous markers of either immature neurons (DCX, PSA-NCAM) or the neurogenic niche (NeuroD, stathmin) in the adult hippocampus, tissue sections were blocked using $10 \%$ horse serum in PB prior to overnight exposure at room temperature to the following antibodies: (1) goat anti-DCX (1:250, Santa Cruz Biotechnology, USA) (2) mouse antiPSA-NCAM (1:500; kind gift from Prof. T. Seki, Juntendo University, Japan; 22) (3) rabbit anti-stathmin (1:250, Calbiochem, USA) (4) goat anti-NeuroD (1:200, Santa-Cruz Biotechnology). Following washes in $0.1 \mathrm{M} \mathrm{PB}$, sections were incubated with secondary antibodies: (1) biotinylated anti-goat IgG (1:250, Vector Laboratories) (2) Alexa 488conjugated donkey anti-mouse (1:250, Molecular Probes, USA) (3) Alexa 488-conjugated donkey anti-rabbit (1:250, Jackson ImmunoResearch, USA) at room temperature for 3 hours. An avidin-biotin complex (Vector Laboratories) was used for signal amplification of biotinylated secondary antibodies, which was then detected with diaminobenzidine (Sigma). To visualize immunofluorescence, sections were mounted in Vectashield (Vector Laboratories) and viewed using a Nikon Eclipse 90i fluorescence microscope.

For experiments to address the neuronal differentiation of adult dentate granule cell progenitors, double-labeling experiments were carried out. Sections were incubated overnight with the following primary antibody cocktails: (1) rat anti-BrdU (1:500, Accurate Biochemicals, USA) with mouse anti-neuronal nuclei (NeuN) (1:1000, Chemicon) to assess the neuronal differentiation of adult hippocampal progenitors, or (2) rat anti-BrdU (1:500, Covance, USA) with rabbit anti-calretinin (1:250, Swant, Switzerland) to examine the colocalization of BrdU with the transient immature neuron marker, calretinin. Sections were then incubated with the following cocktails of secondary antibodies: (1) biotinylated anti-rat $\operatorname{IgG}$ (1:500, Chemicon) with Alexa 555-conjugated anti-mouse (1:250, Molecular Probes) (2) Cy3-conjugated donkey anti-rat and FITC-conjugated donkey anti-mouse (both 1:250, Jackson ImmunoResearch). Signal amplification was performed using FITC-conjugated streptavidin (1:250, Molecular Probes), following which sections were mounted using Vectashield (Vector Laboratories). Sections were observed with a Zeiss Axiovert confocal laser scanning microscope (510LSM) to determine immunofluorescence colocalization using confocal Z-plane sectioning.

For experiments to determine the neuronal or glial differentiation of adult dentate granule cell progenitors, triple-labeling studies were carried out. Sections were incubated overnight 
with a cocktail of primary antibodies: rat anti-BrdU (1:500, Accurate Biochemicals) with mouse anti-NeuN (1:1000, Chemicon) and rabbit anti-glial fibrillary acidic protein (GFAP) (1:500, Chemicon). Sections were incubated with a cocktail of secondary antibodies: biotinylated anti-rat IgG (1:500, Chemicon) with Alexa 555-conjugated anti-mouse (1:250, Molecular Probes) and Cy5-conjugated anti-rabbit (1:500, Chemicon), followed by incubation with Alexa 488-conjugated streptavidin (1:500, Molecular Probes). Sections were mounted using Vectashield (Vector Laboratories), and immunofluorescence colocalization was determined using confocal Z-plane sectioning with an Olympus Fluoview FV 1000 laser scanning microscope.

For double labeling in TRa1-GFP mice, sections were incubated overnight with rabbit antiGFP (1:5000, Abcam, United Kingdom) along with either (1) goat anti-NeuroD (1:200, Santa Cruz Biotechnology) or (2) goat anti-DCX (1:250, Santa Cruz Biotechnology), followed by a cocktail of secondary antibodies: (1) anti-goat Alexa 594 and (2) anti-rabbit Alexa 488 (both 1:1000, Invitrogen, Sweden). For BrdU double-labeling, the sections were first incubated with anti-GFP primary and secondary antibodies before BrdU pre-treatment and immunohistochemistry as described above. The GFP signal was specific as demonstrated by the lack of any immunofluorescence with an anti-GFP antibody in wild type mice. Colocalization of GFP signal in TRa1-GFP mice with the markers (BrdU, NeuroD and DCX) was examined using confocal z-stack images on a Zeiss Axiovert confocal laser scanning microscope (510LSM). For all immunohistochemistry and immunofluorescence experiments the specificity of antibody signal was confirmed using the following controls: (1) absence of primary antibody, or (2) isotype matched IgG controls.

\section{Cell counting}

Quantitation of BrdU-positive cell number in hippocampal sections was carried out using a previously described modified, unbiased stereology protocol (24) on a Zeiss Axioskop microscope. Sections spanned the rostro-caudal extent of the hippocampus (Bregma -1.34 to -3.80) (25) and every 6th hippocampal section was processed for quantitation (12 sections / animal). Sections were coded and the quantitation was conducted by an experimenter blind to the code. BrdU-positive cells within dentate gyrus were counted as being in the subgranular zone (SGZ)/granule cell layer (GCL) when they were directly touching the SGZ or within it. The total number of BrdU-positive cells in the SGZ/GCL was estimated by multiplying the total number of BrdU cells counted from every $6^{\text {th }}$ section by the section periodicity (6).

Quantitation of DCX-positive cells in hippocampal sections was carried out by an experimenter blind to the study code. The number of DCX-positive cells in the SGZ of the DG were quantitated (four sections/animal, $n=5 /$ group). The results were expressed as the number of DCX-positive cells per section. We also addressed the morphological status of DCX-positive cells by categorizing them as (1) DCX-positive cells without tertiary dendrites or (2) DCX-positive cells with complex tertiary arbors (26). Quantitation of both DCXpositive cell number and morphological category was performed under blinded conditions using a Zeiss Axioskop at a magnification of 400X. Quantitation of PCNA, PSA-NCAM, 
stathmin and NeuroD-positive cells was performed using the same approach used for quantitation of DCX-positive cell number.

To examine the differentiation of BrdU-positive progenitors into neurons or glia in the TRa1 -/- and TRa2 -/- mice, the percentage of BrdU-positive cells which colocalized with the neuronal marker NeuN or the glial marker GFAP was determined using confocal microscopy. In each animal ( $\mathrm{n}=5$ /group), $20 \mathrm{BrdU}$-positive cells were analyzed using $\mathrm{Z}$ plane sectioning with $0.41 \mu \mathrm{m}$ steps on an Olympus Fluoview FV100 laser scanning confocal microscope to confirm colocalization with either NeuN or GFAP. In the TRa $1+/ \mathrm{m}$ mice, $50 \mathrm{BrdU}$-positive cells in each animal per marker were analyzed to confirm colocalization with either the transient neuronal marker calretinin or the mature neuronal marker NeuN, using z-plane sectioning on a Zeiss Axiovert 510LSM confocal laser scanning microscope. Percent colocalization of BrdU with either NeuN or calretinin was determined for BrdU-positive cells within the SGZ/GCL or directly in contact with the SGZ and was confirmed using z-plane stack analysis of confocal images.

In TRa1-GFP mice, 20 BrdU, NeuroD, or DCX immunopositive cells per animal ( $\mathrm{n}=3$ ) were analyzed for colocalization with GFP using Z-plane sectioning on a Zeiss Axiovert 510LSM confocal laser scanning microscope.

\section{Statistical analysis}

Results were subjected to statistical analysis using the program Prism (Graphpad, USA). Experiments with two groups were analyzed for differences using the unpaired Student's ttest, with significance determined at $\mathrm{p}<0.05$. Experiments with four groups were subjected to statistical analyses using Analysis of Variance (ANOVA) followed by the Bonferroni post-hoc test, with significance determined at $\mathrm{p}$ values $<0.05$.

\section{Results}

\section{TRa1 is expressed in newborn neurons of the adult hippocampus}

Given recent evidence that thyroid hormone regulates adult hippocampal neurogenesis $(9,10,11)$ and that the most abundant thyroid hormone receptor isoform in the brain is TRa1 (16), we sought to further elucidate the function of this receptor in hippocampal neurogenesis. To examine the expression of the TRa1 receptor in adult hippocampal progenitors, we used a novel knock-in TRa1-GFP mouse strain generated by inserting the coding sequence of eGFP in frame with the TRa1 gene. In the hippocampus, GFP expression was observed within nuclei of cells in the subgranular zone (SGZ) and the granule cell layer (GCL) of the dentate gyrus (DG) subfield. Double immunohistochemistry for the mitotic marker BrdU and GFP was performed to determine if TRa1 is expressed by the proliferating pool of adult hippocampal progenitors. The lack of colocalization of TRa1GFP with BrdU in hippocampal progenitors (Fig. 1A) indicated that this receptor was not expressed by proliferating progenitor cells within the SGZ in the DG. To address if TRa1 is expressed later in neuronal development, i.e. in the largely postmitotic pool of adult hippocampal progenitors, we performed double immunohistochemistry for GFP and two markers for immature neurons; NeuroD and DCX. Confocal analysis revealed that TRa1 
was expressed in both NeuroD and DCX positive progenitors in the SGZ and GCL within the DG. These results indicate that within the neurogenic niche of the adult hippocampus, TRa 1 is predominantly expressed by postmitotic progenitors destined to acquire a neuronal fate, and does not appear to be present in proliferating progenitors. Based on these findings along with our previous results (10), we hypothesized that TRa1 plays a role in the survival of adult hippocampal progenitors, and used mice lacking, or harboring a mutation, in the TRa isoforms to further investigate this hypothesis.

\section{Proliferation of adult hippocampal progenitors is unaffected in the dentate gyrus of TRa1-/- and TRa2-/- mice}

Male TRa1-/- mice and wild type littermate controls were injected with BrdU 2 hours prior to sacrifice in order to assess the influence of TRa1 receptor loss on the proliferation of adult hippocampal progenitors (Fig 2). Stereological analysis indicated no change in the number of BrdU-positive progenitors in the SGZ/GCL of TRa1-/- mice as compared to wild type animals (Fig. 2B). We also examined the expression of an endogenous marker of cell division, proliferating cell nuclear antigen (PCNA), and the number of PCNA-positive cells in the SGZ/GCL was unaltered in TRa1-/- mice (Fig. 2C). We next examined the numbers of BrdU- and PCNA-positive cells in the SGZ/GCL of TRa2-/- mice that overexpress TRa1 as a direct consequence of the gene targeting strategy. TRa2-/- mice do not exhibit any change in the number of BrdU- or PCNA-positive cells within the SGZ/GCL indicating no effect in these mice on hippocampal progenitor proliferation (Fig 2B). In both wild type and TRa1 and TRa2 deficient mice, BrdU and PCNA positive cells were observed in clusters within the SGZ at the border of the GCL and the hilus.

\section{TRa1-/-and TRa2-/- mice show opposing survival of adult hippocampal progenitors}

To examine the postmitotic survival of adult hippocampal progenitors, TRa1-/-and TRa2-I - knockout mice and their respective wild type controls received daily BrdU injections for three days, and were sacrificed 28 days later. TRa1-/- mice showed a significant increase in the number of BrdU-positive cells in the SGZ/GCL as compared to wild type controls which indicated an increased survival of adult hippocampal progenitors in the mutants (Fig 3B). In striking contrast, TRa2-/- mice demonstrated a significant decline in the number of persisting BrdU-positive cells in the SGZ/GCL (Fig 3B). The surviving BrdU-positive cells exhibit a characteristic ovoid shape and are not observed in clusters but are seen dispersed through the granule cell layer (Fig. 3A, C).

\section{TRa1-/- and TRa2-/- mice exhibit contrasting effects on the numbers of DCX-positive immature neurons in the adult dentate gyrus}

DCX is a microtubule-associated protein expressed by post-mitotic and migratory adult hippocampal progenitors (12), and is a useful endogenous marker for adult hippocampal neurogenesis (29). TRa1-/- mice exhibited a significant increase in the number of DCXpositive cells/section within the SGZ/GCL (Fig. 4B), whereas TRa2-/- mice had reduced DCX-positive cell numbers as compared to their wild type controls (Fig 4D). Taken together, the BrdU and DCX results indicate an increased hippocampal neurogenesis in TRa1-/- mice in contrast to the decrease seen in the TRa2-/- strain. 
DCX immunohistochemistry marks both the cell body and the dendritic arbors of newborn neurons (Fig. 4A, C). As immature neurons undergo a morphological maturation, the dendritic tree becomes more elaborate with the presence of complex tertiary dendrites. As described previously (26), we sub-categorized DCX-positive cells as those with or without tertiary dendrites (Fig 4E). While numbers of DCX-positive cells were clearly different in TRa1-/- and TRa2-I- mice, we did not observe any change in the morphological maturation of these DCX-positive cells, as the percentage of DCX-positive cells with complex tertiary dendrites was unaltered in the mutants as compared to their controls (Fig. 4C).

\section{TRa1-/- and TRa2-/- mice show an altered expression of markers of the neurogenic niche}

Immunohistochemical analyses were performed to determine if TRa1-/- and TRa2-/mice exhibit changes in expression of additional stage-specific markers for adult hippocampal neurogenesis, such as PSA-NCAM and stathmin which have previously been shown to co-localize with DCX expression $(13,30)$. Similar to what was seen with DCX, TRa1-/- mice exhibited a significant increase, whereas TRa2-/- mice showed a significant decline in the number of cells positive for PSA-NCAM (TRa1-/-: Fig. 5A, TRa2-/-: Fig. 5B) and stathmin (TRa1-/-: Fig. 5C, TRa2-/-: Fig. 5D) as compared to their wild type controls. While PSA-NCAM expression (Fig. 5A, B) was noted in both the cell body as well as the dendritic arbor similar to DCX expression, stathmin expression (Fig. 5C, D) was predominantly observed in the cell body. Taken together, our results demonstrate that TRa1-/- mice show a significant increase, while TRa2-/- mice exhibit a decline, in the number of DCX-, PSA-NCAM- and stathmin-positive cells within the adult hippocampal neurogenic niche.

We also sought to address whether the TRa1-/- and TRa2-/- mice exhibit any gross changes in hippocampal and granule cell layer volume using modified stereological approaches (10). We observed no significant change in either the total hippocampal volume or in the volume of the granule cell layer in the dentate gyrus hippocampal subfield in the TRa1-/- or TRa2-/- mice as compared to their respective littermate wild type controls (Suppl Fig. 1). Further, granule cell neuron marker (Prox1) expression, mossy fiber bundle innervation of the CA3, and GFAP-immunopositive glial cell numbers in the dentate gyrus subfield were found to be unaltered in the TRa1-/- or TRa2-/- mice as compared to their respective littermate wild type controls (Suppl Fig. 2, Fig 3).

\section{Differentiation of newborn progenitors is not perturbed in TRa1-/- and TRa2-/- mice}

To address whether cell fate acquisition by newborn adult hippocampal progenitors is altered in TRa1-/- and TRa2-/- mice, we examined the percent colocalization of BrdU with the neuronal marker NeuN or the glial marker GFAP, 28 days after BrdU administration using triple immunofluorescence and confocal microscopy. Quantitative analysis, based on confocal $z$-stack analysis to detect colocalization, revealed no change in the percent colocalization of BrdU with NeuN or GFAP in both TRa1-/- and TRa2-/mice as compared to the wild type controls (Fig. 6B, C). In wild type as well as TRa1-/and TRa2-/- mice, more BrdU-positive cells acquired a neuronal, rather than a glial, fate. 
BrdU positive cells that do not colocalize with either NeuN or GFAP may represent as yet undifferentiated cells.

\section{Thyroid hormone administration to TRa2-/- mice rescues the decline in the number of DCX-positive immature neurons}

We hypothesized that the decreased survival of BrdU-positive progenitors and the decline in DCX, PSA-NCAM and stathmin cell numbers in TRa2-/- mice may reflect a limited availability to ligand for the overexpressed TRa1, thus causing an aporeceptor effect in the neurogenic niche similar to those described previously for other tissues (18). To test this hypothesis, we treated the TRa2-/- mice with excess thyroid hormone. Animals were divided into four groups, with TRa2-/- mice and their wild type controls receiving either vehicle or thyroid hormone in drinking water for sixteen days prior to sacrifice. We then assessed whether the hormone treatment could rescue the decrease in number of hippocampal progenitors expressing the endogenous marker, DCX, in the TRa2-/- mice. Thyroid hormone treatment was capable of completely rescuing the decline in DCX-positive cell number in the SGZ/GCL of TRa2-/- mice (Fig 7 B). Interestingly, thyroid hormone treatment also significantly enhanced the number of DCX-positive cells in the SGZ/GCL of wild type animals. These results support the hypothesis that the decrease in progenitor survival observed in TRa2-/- mice, is a consequence of a TRa1 aporeceptor effect, which can be ameliorated through the restoration of an appropriate ligand-receptor ratio via thyroid hormone treatment.

\section{Rescue of survival and neuronal differentiation of adult hippocampal progenitors in TRa1+/m mice by thyroid hormone administration}

To further address the hypothesis that an unliganded TRa1 results in a decline in adult hippocampal neurogenesis, we used mice expressing a mutant TRa1 with dominant negative activity. These heterozygous mice $(\mathrm{TRa} 1+/ \mathrm{m})$ were generated by introducing a point mutation in the TRa1 gene (TRa1R384C) that lowers the affinity for thyroid hormone 10fold (20). We first studied the effects of reduced ligand-binding in TRa1+/m mice on adult hippocampal progenitor proliferation. Wild type controls and TRa1+/m mice received a single BrdU injection and were sacrificed 2 hours later. BrdU immunohistochemistry followed by stereological quantitative analysis showed that mutant mice did not differ from wild type animals in the number of BrdU-positive cells; these results were corroborated by PCNA immunohistochemistry (Fig. 8A, B).

To examine the survival of BrdU-positive progenitors in the hippocampus, wild type controls and TRa $1+/ \mathrm{m}$ mice were injected with BrdU once daily for 3 days and sacrificed 30 days after the last BrdU injection. As compared to wild type mice, a significant decrease in the number of surviving BrdU-positive cells was seen in TRa1+/m mice. Thyroid hormone treatment of TRa $1+/ \mathrm{m}$ mice normalized the numbers of surviving BrdU-positive cells as compared to vehicle treated wild type controls (Fig. 8D). Interestingly, thyroid hormone treatment to wild type controls itself resulted in an increased number of BrdU-positive cells that persisted 30 days post BrdU injection. Taken together with the results obtained from the TRa2-I- mice, the data indicates that an unliganded TRa1 receptor could function as an 
inhibitor of adult hippocampal neurogenesis through an effect on the survival of newborn hippocampal progenitors.

To determine if the unliganded TRa1 influences the differentiation of BrdU-positive progenitors into mature neurons, double-labeling studies were performed using antibodies to BrdU and NeuN. Confocal analysis revealed a significant reduction in the percentage of BrdU-positive cells that are immunopositive for the neuronal marker NeuN (Fig. 8E) indicating that fewer progenitor cells acquire a neuronal phenotype in TRa $1+/ \mathrm{m}$ mice. Thyroid hormone treatment ameliorated the decrease in neuronal differentiation observed in TRa1+/m mutant mice (Fig. 8E). However, thyroid hormone treatment to wild type mice did not influence neuronal differentiation. These results suggest that unliganded TRa1 influences both hippocampal progenitor cell survival and neuronal differentiation.

Given the decline observed in BrdU/NeuN colocalization in TRa1+/m mice, we next sought to address whether the expression of calretinin in BrdU-positive postmitotic hippocampal progenitors is also altered in TRa $1+/ \mathrm{m}$ mice. Calretinin, a calcium binding protein, is transiently expressed in the postmitotic stages of hippocampal progenitor cell development (31), and calretinin-immunopositive cells represent a fraction of immature, postmitotic adult hippocampal progenitors that are destined for neuronal differentiation. Calretinin expression is thought to precede the expression of the mature neuronal marker NeuN. Confocal analysis revealed a significant decline in the colocalization of BrdU with calretinin in the TRa $1+/ \mathrm{m}$ mice, which was partially normalized by thyroid hormone treatment (Fig. 8F).

\section{Decrease in NeuroD positive cells in the hippocampal neurogenic niche of TRa2-/- and TRa 1+/m mice}

The transcription factor NeuroD is required for the differentiation of dentate granule cells of the hippocampus (32), and is expressed in adult hippocampal progenitors $(33,34)$. We examined by immunohistochemistry the number of NeuroD-positive cells within the SGZ/GCL of TRa2-/- and TRa1+/m mice. In wild type mice, NeuroD was strongly expressed in the SGZ where adult hippocampal progenitors reside, whereas the expression was substantially lower in the GCL (Fig. 9A, C). In both mutant mouse strains we observed a significant reduction in the number of NeuroD-immunopositive cells within the SGZ/GCL as compared to their respective wild type controls (Fig 9). Thyroid hormone treatment normalized the number of NeuroD-positive cells in TRa1+/m mice (Fig. 9D). In the TRa2-/ - mice, thyroid hormone treatment showed a trend to increase the number of NeuroDpositive cells/section, however this did not reach significance $(p=0.06$, ANOVA and Bonferroni post-hoc test) (Fig. 9B). Interestingly, thyroid hormone treatment resulted in a significant increase in NeuroD-positive cell number in wild type control littermates from both the TRa2-/- and TRa1+/m experiments (Fig 9B, D).

\section{Discussion}

Our aim in this study was to address the role of TRa1 in adult hippocampal neurogenesis using TRa1-/-, TRa2-/- and TRa1+/m mutant mice. We provide novel evidence that TRa1 regulates adult hippocampal neurogenesis, through effects on progenitor survival and neuronal differentiation. The striking similarity between the decreased adult neurogenesis 
observed in hypothyroidism $(9,10)$ and our results of the neurogenic phenotype in mutant mice with TRa 1 aporeceptor activity, strongly suggest that an unliganded TRa1 contributes to the neurogenic decline in adult-onset hypothyroidism.

While the requirement for thyroid hormone during neurodevelopment is well established $(35,36)$, it is only recently that the effects of thyroid hormone on adult neurogenesis have been reported $(9,10,11,27)$. Impaired adult hippocampal neurogenesis is implicated as a key structural correlate that contributes to the functional deficits in hippocampal dependentlearning and mood-related behaviors observed in rodents and humans with adult-onset hypothyroidism $(4,11,37)$. However, the role of individual TRs in adult neurogenesis remains largely unidentified, with a single report (27) suggesting a role for TRa isoforms in the regulation of progenitor cell cycling in the subventricular zone lining the lateral ventricles.

Approximately $75 \%$ of thyroid hormone binding by TRs in the adult rodent brain is accounted for by TRa $1(16,38)$. Immunohistochemical evidence indicates TRa isoform expression in adult hippocampal progenitors (10), but their stage-specific expression is unknown. Our colocalization studies of the TRa1-GFP chimeric protein with stage-specific progenitor markers indicate TRa 1 expression in NeuroD and DCX immunopositive progenitors, but not in proliferating BrdU-positive progenitors. Our unpublished results with the TRa1-GFP knock-in mice indicate that TRa1 expression is restricted to postmitotic stages of neuronal progenitor development (Wallis et al., manuscript in press). Further, proliferation was not altered in TRa1-/- mice, TRa2-/- mice or TRa1+/m mutant mice. Taken together, these results imply that neither TRa 1 nor TRa 2 influence adult hippocampal progenitor proliferation. This is interesting in light of previous evidence $(9,10)$ that indicate no change in progenitor turnover following hypo- or hyperthyroidism, suggesting a role for thyroid hormone in the postmitotic stages of adult hippocampal progenitor development.

TRa1-/- mice exhibited an increased survival of adult hippocampal progenitors, in striking contrast to the TRa1-overexpressing TRa2-/- mice that show a significant decline in progenitor survival. It could be argued that the decrease in postmitotic progenitor survival in the TRa2-/- mice is a consequence of TRa1 overexpression, or TRa2 loss, or a combination of both. However, we hypothesized, based on the previously described phenotype of the TRa2-/- mice (18) that the overexpression of TRa1 may result in aporeceptor activity due to limited availability to ligand, and therefore reduced postmitotic survival of adult hippocampal progenitors. Strong support for this hypothesis comes from our results with the TRa1+/m mice that express a mutant TRa1 with aporeceptor activity, which show a robust decline in adult hippocampal progenitor survival. Both the TRa2-/and TRa $1+/ \mathrm{m}$ mice show a phenotype similar to hypothyroidism with decreased hippocampal progenitor survival, adding credence to the hypothesis that a TRa1 aporeceptor contributes to the deleterious consequences of hypothyroidism on adult hippocampal neurogenesis (Fig. 10). Interestingly, TRa 1 deficiency enhances hippocampal progenitor survival suggesting the possibility that in the TRa1-/-mice, besides the loss of liganded TRa1, the removal of a strong repressor activity of unliganded TRa1 may underlie the increased progenitor survival. 
The cellular mechanisms for the effects of TRa 1 on hippocampal progenitor survival are at present unclear. However, it is tempting to draw parallels with the proposed mechanisms for the actions of TRa1 on embryonic neuronal progenitors. Unliganded TRa1 has been suggested to arrest neuronal progenitors at specific stages of development until ligand availability allows them to progress further into neuronal differentiation (39). Adult hippocampal progenitors may recapitulate the effects of TRa1 aporeceptors observed in their developmental counterparts, with the TRa 1 aporeceptor affecting expression of genes relevant for the progression of adult hippocampal progenitors to the next developmental stage. Indeed in both the TRa2-/- and TRa $1+/ \mathrm{m}$ mice, the decline in progenitor survival suggests that TRa1 aporeceptors may result in postmitotic progenitors being arrested during maturation, with an eventual cell loss in the absence of a rescue by the ligand. Further support for this idea comes from our observations that thyroid hormone administration to TRa2-/- and TRa1+/m mice rescues the decreased survival observed using either an endogenous marker (DCX) or exogenous label (BrdU). The data also indicate that thyroid hormone treatment to wild type mice increases hippocampal progenitor survival. Our results lead us to speculate that the effects of thyroid hormone on adult hippocampal neurogenesis may largely involve an alleviation of the TRa1 aporeceptor effect. A question that remains at present is whether the actions of TRa1, either liganded or unliganded, on adult hippocampal progenitor survival involve cell autonomous or non-cell autonomous effects via modulation of the neurogenic niche. We have previously shown that thyroid hormone does exert direct effects on hippocampal progenitors in vitro (10), however the in vitro effects differ substantially from the effects of perturbed thyroid hormone levels in vivo, indicating that dispersed progenitor cultures do not recapitulate the in vivo neurogenic niche vis a vis responses to thyroid hormone. Future studies that address the influence of TRa1 on neurogenic niche trophic factor expression, deiodinase expression in the hippocampal neurogenic niche and on astrocytic release of regulatory factors would further our mechanistic understanding of the effects of TRa 1 on hippocampal neurogenesis.

Adult-onset hypothyroidism, besides effects on hippocampal progenitor survival also significantly decreases neuronal differentiation, an effect relieved by thyroid hormone treatment $(9,10)$. The TRa $1+/ \mathrm{m}$ mice exhibit a significant decline in the survival and neuronal differentiation of hippocampal progenitors, both of which can be rescued by thyroid hormone. This strong phenotypic similarity suggests that unliganded TRa1 may account for all the changes observed in hippocampal neurogenesis in hypothyroidism. However, it is important to note that TRa2-/- mice, that overexpress TRa1, despite a robust decline in progenitor survival show normal neuronal differentiation of hippocampal progenitors, indicating a difference from the TRa $1+/ \mathrm{m}$ mice. This discrepancy is likely to be due to the weak aporeceptor activity of TRa1 in the TRa2-/- mice, as caused by the receptor overexpression, as compared to the relatively much stronger aporeceptor activity caused by the point mutation in the TRa1R384C mutant receptor. Other contributing factors may include the differences in TR stoichiometry in these mutant mice. Future studies addressing the regulation of key target genes that modulate hippocampal progenitor survival and neuronal differentiation may help to resolve these issues. The TRa1-/- mice that show enhanced hippocampal progenitor survival exhibit normal neuronal differentiation, 
suggesting that deficiency of this receptor may not be capable of further enhancing the neuronal differentiation of adult hippocampal progenitors.

We further addressed the early stages of postmitotic hippocampal progenitor development using two different markers, calretinin and NeuroD. Calretinin is a transient marker of postmitotic hippocampal progenitors destined for neuronal differentiation (31). The TRa1+/m mice show decreased BrdU-calretinin percent colocalization, which can be rescued by thyroid hormone. Thyroid hormone administration to wild type mice enhances the percentage of BrdU labeled progenitors that are calretinin-immunopositive. Since we observed an influence of TRa 1 aporeceptors on the calretinin positive stage of neuronal differentiation in adult hippocampal progenitors, we examined whether an earlier stage of fate choice namely the acquisition of neuronal fate through the transcription factor NeuroD is also influenced. We observed a robust decline in NeuroD positive cell numbers within the hippocampal neurogenic niche of both TRa1+/m and TRa2-/- mice, which could be rescued by thyroid hormone administration. NeuroD is expressed in adult hippocampal progenitor cells (33) and is essential for progenitor survival and neuronal differentiation (32, 40). Furthermore, NeuroD expression is regulated by thyroid hormone, and hypothyroidism reduces NeuroD expression in the developing rat cerebellum (41) and the adult hippocampus (unpublished results). There are strong parallels to the effects of thyroid hormone on myogenic differentiation, where the TRa 1 aporeceptor represses the transcription of the myogenic transcription factor, MyoD (42). Our results suggest that TRa1 aporeceptor activity may hold hippocampal progenitors at the early postmitotic stage prior to the acquisition of NeuroD-positive identity and the commitment to a neuronal fate. The ligand thyroid hormone could act to allow progression of neuronal differentiation, and based on reports that thyroid hormone cooperates with neurotrophins during hippocampal development (43) may also modulate the sensitivity of these postmitotic progenitors to neurotrophins within the niche.

Previous reports demonstrate that TRa1+/m mice also exhibit depressive behavior (44), anxiety and memory impairments that are rescued by thyroid hormone treatment (21). Given the role of adult hippocampal neurogenesis in hippocampal-dependent memory $(7,45)$, depression (8) and anxiety behavior (46), this highlights the possibility that decreased hippocampal neurogenesis as a consequence of TRa1 aporeceptor activity may contribute to the behavioral deficits observed in both hypothyroidism and TRa1+/m mutant mice. In contrast, the TRa1-/- mice exhibit enhanced fear associated learning, a behavior known to require hippocampal neurogenesis (47). The increased neurogenesis we observed in the TRa1-/- mice may contribute to the improved fear learning. However, the TRa1-/- mice also exhibit enhanced anxiety behavior, which has a more controversial association with neurogenesis, with increased anxiety behavior observed both following a decline or induction in hippocampal neurogenesis $(48,49)$. Our results motivate studies to determine the contribution of the neurogenic changes observed in the TRa1 mutants to the behavioral phenotypes observed in these mice.

In conclusion, we provide definitive evidence that TRa 1 regulates adult hippocampal neurogenesis. Further, our data strongly indicate that TRa 1 aporeceptor activity mediates the impaired survival and neuronal differentiation of adult hippocampal progenitors in adult- 
onset hypothyroidism. Our results highlight the requirement for future studies to identify the target genes that are regulated by both TRa1 apo- and holo-receptor activity, and their contribution to the decreased hippocampal neurogenesis and cognitive/behavioral deficits observed in adult-onset hypothyroidism.

\section{Supplementary Material}

Refer to Web version on PubMed Central for supplementary material.

\section{Acknowledgments}

This work was supported by intramural funds from TIFR and a Wellcome Trust Senior Overseas Fellowship in Biomedical sciences to VV (04082003114133). Support to BV was obtained from the Swedish Research Council, the Swedish Cancer Society and the Wallenberg Foundations. MvH received a Swedish Society for Medical Research (SSMF) fellowship. We are grateful to Professor T. Seki, Juntendo University School of Medicine, Tokyo, for the gift of the PSA-NCAM antibody.

\section{References}

1. Bernal J. Action of thyroid hormone in brain. J Endocrinol Invest. 2002; 25:268-88. [PubMed: 11936472]

2. Bernal J. Thyroid hormone receptors in brain development and function. Nat Clin Pract Endocrinol Metab. 2007; 3:249-259. [PubMed: 17315033]

3. Osterweil D, Syndulko K, Cohen SN, Pettler-Jennings PD, Hershman JM, Cummings JL, Tourtellotte WW, Solomon DH. Cognitive function in non-demented older adults with hypothyroidism. J Am Geriatr Soc. 1992; 40:325-335. [PubMed: 1556359]

4. Dugbartey AT. Neurocognitive aspects of hypothyroidism. Arch Intern Med. 1998; 158:1413-1418. [PubMed: 9665349]

5. Fundaro A. Behavioral modifications in relation to hypothyroidism and hyperthyroidism in adult rats. Prog Neuropsychopharmacol Biol Psychiatry. 1989; 13:927-940. [PubMed: 2813810]

6. Kulikov A, Torrésani J, Jeanningros R. Experimental hypothyroidism increases immobility in rats in the forced swim paradigm. Neurosci Lett. 1997; 234:111-114. [PubMed: 9364510]

7. Kitabatake Y, Sailor KA, Ming GL, Song H. Adult neurogenesis and hippocampal memory function: new cells, more plasticity, new memories? Neurosurg Clin N Am. 2007; 18:105-113. [PubMed: 17244558]

8. Vaidya VA, Fernandes K, Jha S. Regulation of adult hippocampal neurogenesis: relevance to depression. Expert Rev Neurother. 2007; 7:853-864. [PubMed: 17610392]

9. Ambrogini P, Cuppini R, Ferri P, Mancini C, Ciaroni S, Voci A, Gerdoni E, Gallo G. Thyroid hormones affect neurogenesis in the dentate gyrus of adult rat. Neuroendocrinology. 2005; 81:244253. [PubMed: 16113586]

10. Desouza LA, Ladiwala U, Daniel SM, Agashe S, Vaidya RA, Vaidya VA. Thyroid hormone regulates hippocampal neurogenesis in the adult rat brain. Mol Cell Neurosci. 2005; 29:414-426. [PubMed: 15950154]

11. Montero-Pedrazuela A, Venero C, Lavado-Autric R, Fernández-Lamo I, García-Verdugo JM, Bernal J, Guadaño-Ferraz A. Modulation of adult hippocampal neurogenesis by thyroid hormones: implications in depressive-like behavior. Mol Psychiatry. 2006; 11:361-371. [PubMed: 16446739]

12. Ming GL, Song H. Adult neurogenesis in the mammalian central nervous system. Annu Rev Neurosci. 2005; 28:223-250. [PubMed: 16022595]

13. Jin K, Mao XO, Cottrell B, Schilling B, Xie L, Row R.H,, Sun, Y. Peel A, Childs J, Gendeh G, Gibson BW, Greenberg DA. Proteomic and immunochemical characterization of a role for stathmin in adult neurogenesis. FASEB J. 2004; 18:287-299. [PubMed: 14769823]

14. Kempermann G, Jessberger S, Steiner B, Kronenberg G. Milestones of neuronal development in the adult hippocampus. Trends Neurosci. 2004; 27:447-452. [PubMed: 15271491] 
15. Yen PM. Physiological and molecular basis of thyroid hormone action. Physiol Rev. 2001; 81:1097-1142. [PubMed: 11427693]

16. Schwartz HL, Strait KA, Ling NC, Oppenheimer JH. Quantitation of rat tissue thyroid hormone binding receptor isoforms by immunoprecipitation of nuclear triiodothyronine binding capacity. $\mathrm{J}$ Biol Chem. 1992; 267:11794-11799. [PubMed: 1601852]

17. Koenig RJ, Lazar MA, Hodin RA, Brent GA, Larsen PR, Chin WW, Moore DD. Inhibition of thyroid hormone action by a non-hormone binding cerbA protein generated by alternative mRNA splicing. Nature. 1989; 337:659-661. [PubMed: 2537467]

18. Saltó C, Kindblom JM, Johansson C, Wang Z, Gullberg H, Nordström K, Mansén A, Ohlsson C, Thorén P, Forrest D, Vennström B. Ablation of TRalpha2 and a concomitant overexpression of alpha1 yields a mixed hypo- and hyperthyroid phenotype in mice. Mol Endocrinol. 2001; 15:2115-2128. [PubMed: 11731613]

19. Wikström L, Johansson C, Saltó C, Barlow C, Campos Barros A, Baas F, Forrest D, Thorén P, Vennström B. Abnormal heart rate and body temperature in mice lacking thyroid hormone receptor alpha 1. EMBO J. 1998; 17:455-461. [PubMed: 9430637]

20. Tinnikov A, Nordström K, Thorén P, Kindblom JM, Malin S, Rozell B, Adams M, Rajanayagam O, Pettersson S, Ohlsson C, Chatterjee K, Vennström B. Retardation of post-natal development caused by a negatively acting thyroid hormone receptor alpha1. EMBO J. 2002; 21:5079-5087. [PubMed: 12356724]

21. Venero C, Guadaño-Ferraz A, Herrero AI, Nordström K, Manzano J, de Escobar GM, Bernal J, Vennström B. Anxiety, memory impairment, and locomotor dysfunction caused by a mutant thyroid hormone receptor alpha1 can be ameliorated by T3 treatment. Genes Dev. 2005; 19:21522163. [PubMed: 16131613]

22. Seki T, Arai Y. Highly polysialylated neural cell adhesion molecule (NCAM-H) is expressed by newly generated granule cells in the dentate gyrus of the adult rat. J Neurosci. 1993; 13:23512358. [PubMed: 7684771]

23. Paxinos, G.; Watson, C. The Rat Brain in Stereotaxic Coordinates. Academic Press; 1998.

24. Malberg JE, Eisch AJ, Nestler EJ, Duman RS. Chronic antidepressant treatment increases neurogenesis in adult rat hippocampus. J Neurosci. 2000; 20:9104-9110. [PubMed: 11124987]

25. Paxinos, G.; Franklin, KBJ. The Mouse Brain in Stereotaxic Coordinates. Academic Press; USA: 2004.

26. Wang JW, David DJ, Monckton JE, Battaglia F, Hen R. Chronic fluoxetine stimulates maturation and synaptic plasticity of adult-born hippocampal granule cells. J Neurosci. 2008; 28:1374-1384. [PubMed: 18256257]

27. Lemkine GF, Raj A, Alfama G, Turque N, Hassani Z, Alegria-Prévot O, Samarut J, Levi G, Demeneix BA. Adult neural stem cell cycling in vivo requires thyroid hormone and its alpha receptor. FASEB J. 2005; 19:863-865. [PubMed: 15728663]

28. Macchia PE, Takeuchi Y, Kawai T, Cua K, Gauthier K, Chassande O, Seo H, Hayashi Y, Samarut J, Murata Y, Weiss RE, Refetoff S. Increased sensitivity to thyroid hormone in mice with complete deficiency of thyroid hormone receptor alpha. Proc. Natl. Acad. Sci. USA. 2001; 98:349-354. [PubMed: 11120878]

29. Couillard-Despres S, Winner B, Schaubeck S, Aigner R, Vroemen M, Weidner N, Bogdahn U, Winkler J, Kuhn HG, Aigner L. Doublecortin expression levels in adult brain reflect neurogenesis. Eur J Neurosci. 2005; 21:1-14. [PubMed: 15654838]

30. Nacher J, Crespo C, McEwen BS. Doublecortin expression in the adult rat telencephalon. Eur J Neurosci. 2001; 14:629-644. [PubMed: 11556888]

31. Brandt MD, Jessberger S, Steiner B, Kronenberg G, Reuter K, Bick-Sander A, von der Behrens W, Kempermann G. Transient calretinin expression defines early postmitotic step of neuronal differentiation in adult hippocampal neurogenesis of mice. Mol Cell Neurosci. 2003; 24:603-613. [PubMed: 14664811]

32. Liu M, Pleasure SJ, Collins AE, Noebels JL, Naya FJ, Tsai MJ, Lowenstein DH. Loss of BETA2/ NeuroD leads to malformation of the dentate gyrus and epilepsy. Proc Natl Acad Sci U S A. 2000; 97:865-870. [PubMed: 10639171] 
33. Seki T. Expression patterns of immature neuronal markers PSA-NCAM, CRMP-4 and NeuroD in the hippocampus of young adult and aged rodents. J Neurosci Res. 2002; 70:327-334. [PubMed: 12391592]

34. Hodge RD, Kowalczyk TD, Wolf SA, Encinas JM, Rippey C, Enikolopov G, Kempermann G, Hevner RF. Intermediate progenitors in adult hippocampal neurogenesis: Tbr2 expression and coordinate regulation of neuronal output. J Neurosci. 2008; 28:3707-3717. [PubMed: 18385329]

35. Calzà L, Aloe L, Giardino L. Thyroid hormone-induced plasticity in the adult rat brain. Brain Res Bull. 1997; 44:549-557. [PubMed: 9370223]

36. Gilbert ME, Paczkowski C. Propylthiouracil (PTU)-induced hypothyroidism in the developing rat impairs synaptic transmission and plasticity in the dentate gyrus of the adult hippocampus. Brain Res Dev Brain Res. 2003; 145:19-29.

37. Baldini IM, Vita A, Mauri MC, Amodei V, Carrisi M, Bravin S, Cantalamessa L. Psychopathological and cognitive features in subclinical hypothyroidism. Prog Neuropsychopharmacol Biol Psychiatry. 1997; 21:925-935. [PubMed: 9380789]

38. Ercan-Fang S, Schwartz HL, Oppenheimer JH. Isoform-specific 3,5,3'-triiodothyronine receptor binding capacity and messenger ribonucleic acid content in rat adenohypophysis: effect of thyroidal state and comparison with extrapituitary tissues. Endocrinology. 1996; 137:3228-3233. [PubMed: 8754744]

39. Muñoz A, Wrighton C, Seliger B, Bernal J, Beug H. Thyroid hormone receptor/c-erbA: control of commitment and differentiation in the neuronal/chromaffin progenitor line PC12. J Cell Biol. 1993; 121:423-438. [PubMed: 8385673]

40. Gao Z, Ure K, Ables JL, Lagace DC, Nave KA, Goebbels S, Eisch AJ, Hsieh J. Neurod1 is essential for the survival and maturation of adult-born neurons. Nat Neurosci. 2009; 12:10901092. [PubMed: 19701197]

41. Chantoux F, Francon J. Thyroid hormone regulates the expression of NeuroD/BHF1 during the development of rat cerebellum. Mol Cell Endocrinol. 2002; 194:157-163. [PubMed: 12242038]

42. Daury L, Busson M, Casas F, Cassar-Malek I, Wrutniak-Cabello C, Cabello G. The triiodothyronine nuclear receptor c-ErbAalpha1 inhibits avian MyoD transcriptional activity in myoblasts. FEBS Lett. 2001; 508:236-240. [PubMed: 11718722]

43. Clos J, Legrand C. An interaction between thyroid hormone and nerve growth factor promotes the development of hippocampus, olfactory bulbs and cerebellum: a comparative biochemical study of normal and hypothyroid rats. Growth Factors. 1990; 3:205-220. [PubMed: 2245042]

44. Pilhatsch M, Winter C, Nordström K, Vennström B, Bauer M, Juckel G. Increased depressive behaviour in mice harboring the mutant thyroid hormone receptor alpha 1. Behav Brain Res. May 16.2010 [Epub ahead of print].

45. Becker S, Wojtowicz JM. A model of hippocampal neurogenesis in memory and mood disorders. Trends Cogn Sci. 2007; 11:70-76. [PubMed: 17174137]

46. Santarelli L, Saxe M, Gross C, Surget A, Battaglia F, Dulawa S, Weisstaub N, Lee J, Duman R, Arancio O, Belzung C, Hen R. Requirement of hippocampal neurogenesis for the behavioral effects of antidepressants. Science. 2003; 301:805-809. [PubMed: 12907793]

47. Guadaño-Ferraz A, Benavides-Piccione R, Venero C, Lancha C, Vennström B, Sandi C, DeFelipe J, Bernal J. Lack of thyroid hormone receptor alpha1 is associated with selective alterations in behavior and hippocampal circuits. Mol Psychiatry. 2003; 8:30-38. [PubMed: 12556906]

48. Jessberger S, Clark RE, Broadbent NJ, Clemenson GD Jr. Consiglio A, Lie DC, Squire LR, Gage FH. Dentate gyrus-specific knockdown of adult neurogenesis impairs spatial and object recognition memory in adult rats. Learn Mem. 2009; 16:147-54. [PubMed: 19181621]

49. Fuss J, Ben Abdallah NM, Vogt MA, Touma C, Pacifici PG, Palme R, Witzemann V, Hellweg R, Gass P. Voluntary exercise induces anxiety-like behavior in adult C57BL/6J mice correlating with hippocampal neurogenesis. Hippocampus. 2010; 20:364-76. [PubMed: 19452518] 

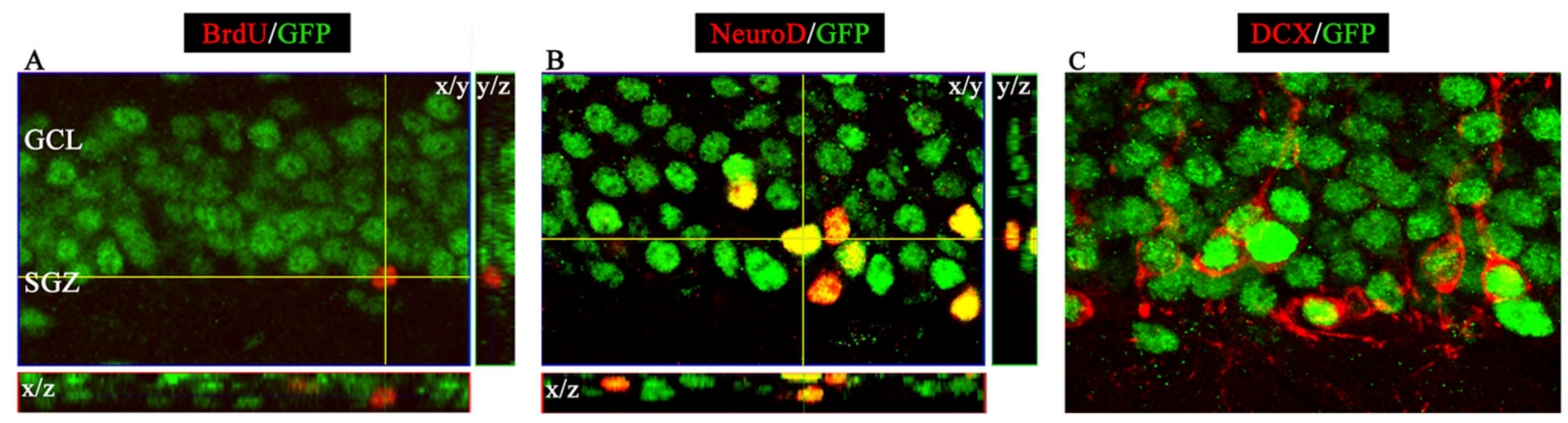

Figure 1.

TRa1 is expressed in Neuro-D and DCX positive progenitors within the dentate gyrus.

TRa1-GFP knock-in mice generated by insertion of the coding sequence of eGFP in frame with the TRa1 gene were utilized to study the expression of TRa1-GFP chimeric protein using double immunoflourescence studies. TRa1-GFP knock-in mice received a single injection of BrdU (150 mg/kg, $\mathrm{n}=3)$ and were sacrificed 2 hours later. (A) Shown is a confocal z-stack image at 630x magnification indicating the lack of colocalization of TRa1GFP with BrdU within the subgranular zone (SGZ) of the dentate gyrus. (B) NeuroDpositive progenitors within the SGZ and granule cell layer (GCL) of the dentate gyrus colocalized with GFP as shown in a representative confocal z-stack image $(630 \mathrm{x}$ magnification). (C) Doublecortin (DCX) immunopositive immature neurons were also observed to exhibit colocalization with GFP at 630x magnification indicating the presence of TRa1 in DCX-positive immature neurons. 

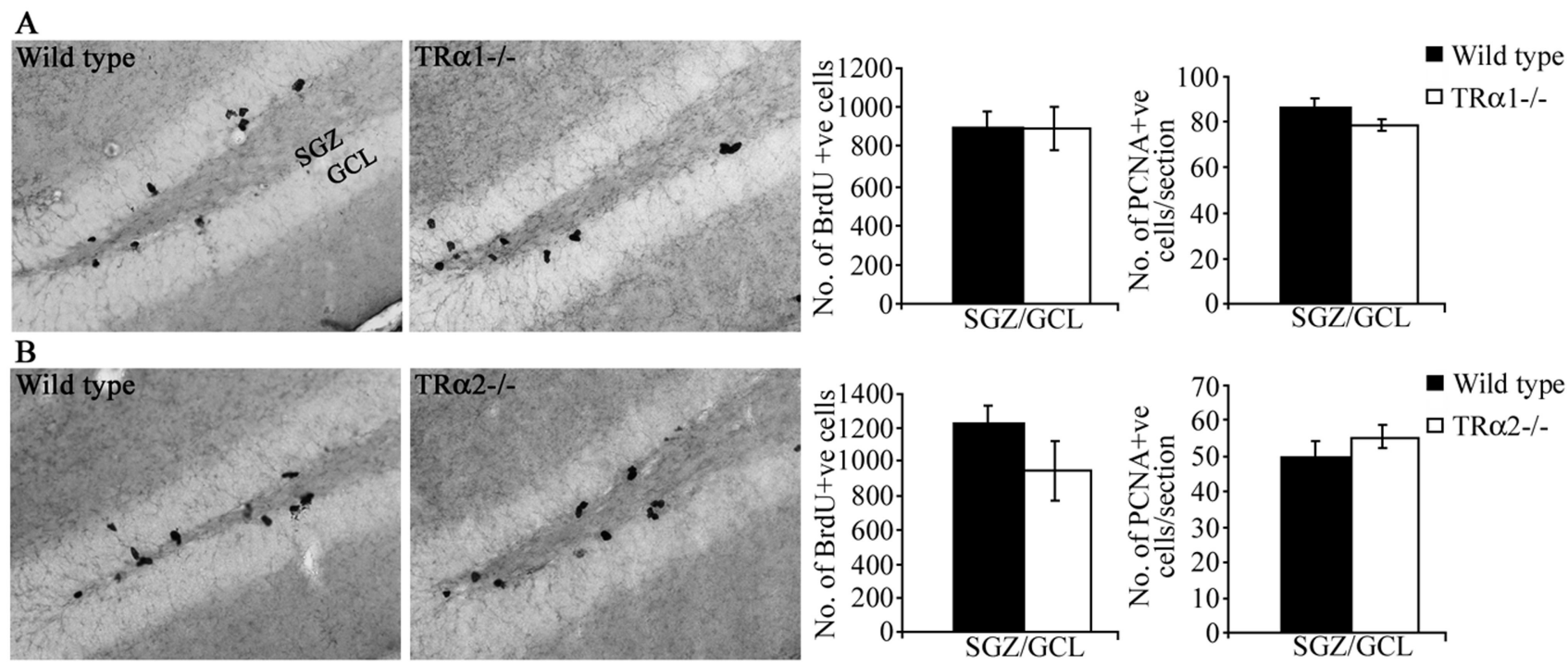

Figure 2.

TRa1 -/- and TRa2-/- mice do not exhibit any change in the proliferation of adult hippocampal progenitors. TRa1-/- and TRa2-/- mice along with their respective wild type littermate controls received a single injection of BrdU (100 mg/kg, $n=3-5 /$ group) and were sacrificed 2 hours later to assess the numbers of proliferating adult hippocampal progenitors in these mutant mice. Shown are representative photomicrographs of BrdU-positive cells at 200x magnification from TRa1-/- (A) and TRa2-/- (D) mice along with their respective wild type controls (A, D). BrdU-positive cells were predominantly observed in clusters within the subgranular zone (SGZ), at the border of the hilus and the granule cell layer (GCL). Stereological analysis revealed no change in the number of BrdU-positive cells within the SGZ/GCL in TRa1-/- (B) and TRa2-/- (E) mice as compared to their respective wild type controls. To examine ongoing adult hippocampal progenitor proliferation we also used immunohistochemical analysis of an endogenous marker of cell proliferation, Proliferating Cell Nuclear Antigen (PCNA). Quantitative analysis did not indicate any change in the numbers of PCNA-positive cells within the SGZ/GCL of TRa1-/ - (C) and TRa2-I- (F) mice as compared to their respective wild type controls. The results are expressed as the mean \pm SEM ( $n=3-5$ / group) number of BrdU-positive cells in the SGZ/GCL, or number of PCNA-positive cells per section. 
A

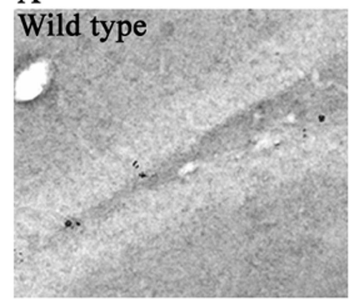

B

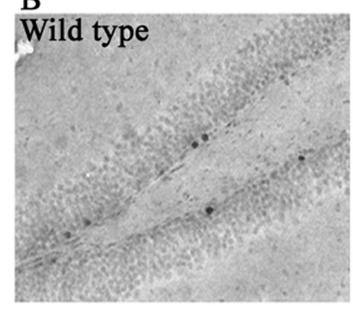

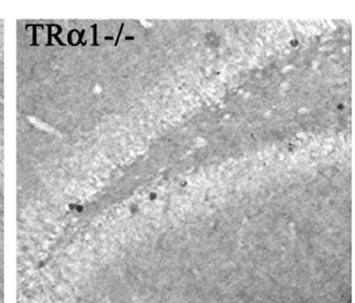

TR $\alpha 2-/-$

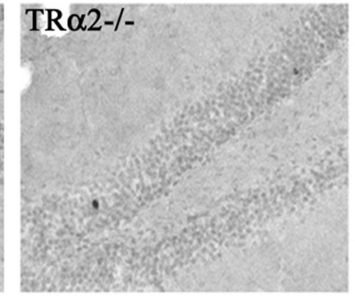

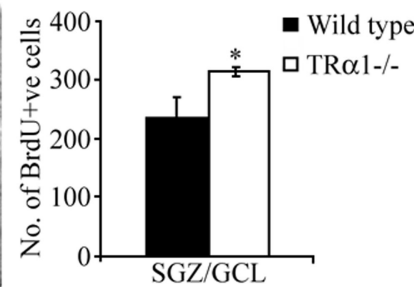

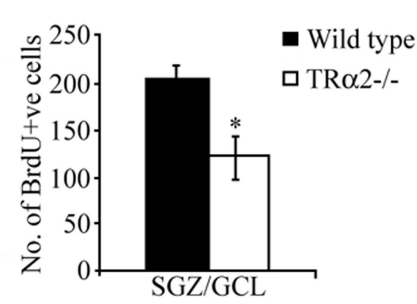

Figure 3.

TRa1-/- mice exhibit an increase in the survival of BrdU-positive adult hippocampal progenitors, whereas TRa2-/- mice show a decrease in the survival of BrdU-positive progenitors. TRa1-/- and TRa2-/- mice, along with their respective wild type littermate controls, received $\mathrm{BrdU}(150 \mathrm{mg} / \mathrm{kg})$ once daily by intraperitoneal injection for 3 consecutive days and were sacrificed 28 days after the final BrdU injection. Shown are representative photomicrographs of BrdU-positive cells from TRa1-/- (A) and TRa2-/(C) mice, along with their respective wild type littermate controls (A, C) at 200x magnification. BrdU-positive cells within the subgranular zone (SGZ) and the granule cell layer (GCL) were ovoid in morphology and rarely observed in clusters. Quantitative stereological analysis showed that the TRa1-/- knockout mice had significantly higher numbers of surviving BrdU-positive cells in the SGZ/GCL (B), whereas the TRa2-/knockout mice showed a significant decrease in the numbers of BrdU-positive cells in the SGZ/GCL (D). The results are expressed as the mean \pm SEM ( $n=3-5$ / group) number of BrdU-positive cells in the SGZ/GCL. *p $<0.05$ indicates significantly different from wild type control (Student's t-test). 
A

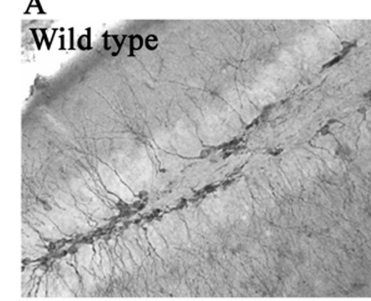

B

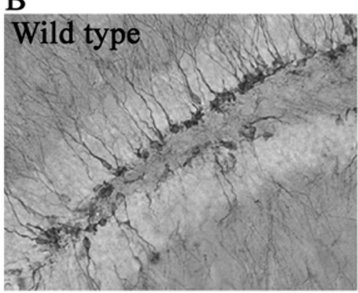

C

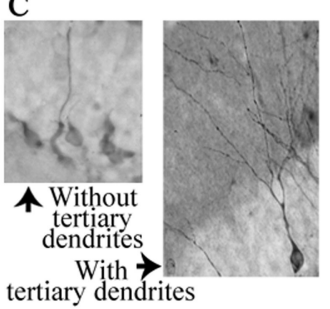

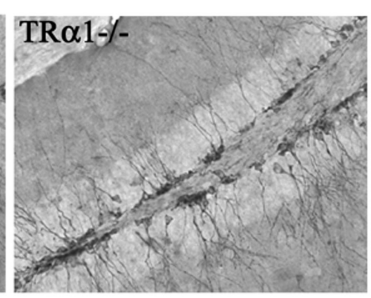
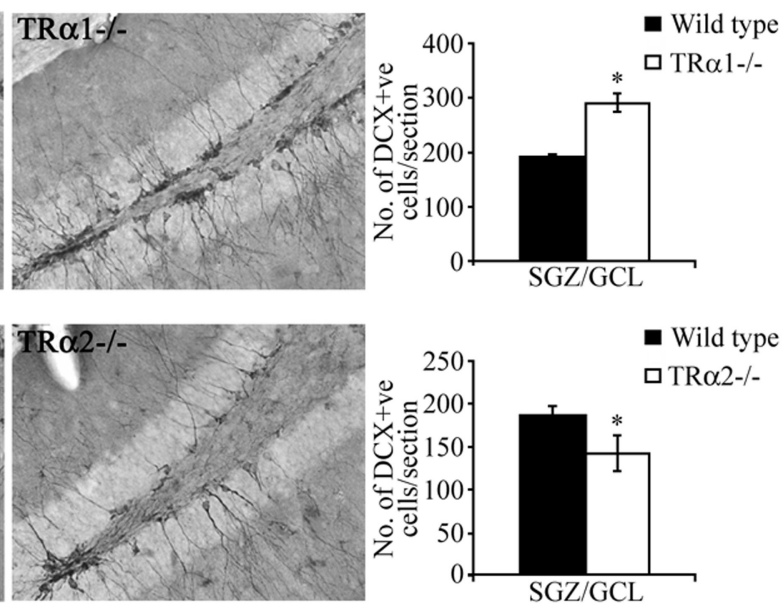

SGZ/GCL

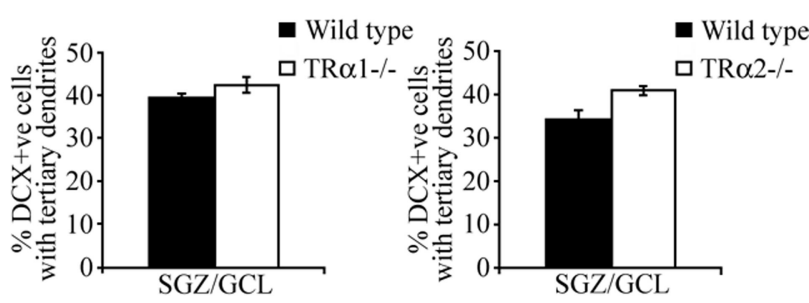

Figure 4.

TRa1-/- mice exhibit an increase in the number of DCX-positive immature neurons in the adult hippocampus, whereas TRa2-/- mice show a decline in the numbers of hippocampal DCX-positive immature neurons. Immunohistochemistry for DCX was carried out to examine the survival of postmitotic adult hippocampal progenitors. Shown are representative photomicrographs of DCX-positive immature neurons at 200x magnification within the subgranular zone (SGZ)/ granular cell layer (GCL) in the dentate gyrus subfield in TRa1-/- (A) and TRa2-/- (C) mice, along with their respective wild type controls (A, C). Quantitative analysis revealed that TRa1-/- mice exhibit a significant increase in the number of DCX-positive cells in the SGZ/GCL as compared to their wild type controls (B). In striking contrast, TRa2-/- mice showed a significant decline in the number of DCXpositive cells in the SGZ/GCL as compared to their wild type controls (D). Analysis of the dendritic morphology of DCX-positive cells revealed no change in the morphological maturation of DCX-positive cells in TRa1-/- and TRa2-/- mice as compared to their wild type littermate controls. Shown are representative images of DCX-positive cells at 400x magnification categorized as with, or without, tertiary dendrites (E). Quantitative analysis of the percent distribution of DCX positive cells with tertiary dendrites in the SGZ/GCL revealed no change in TRa1-/- and TRa2-/- mice as compared to wild type controls (F, $\mathrm{G})$. The results are expressed as the mean \pm SEM ( $n=3$-5/group) number of DCX-positive cells per section in the SGZ/GCL, or mean \pm SEM ( $n=3-5 /$ group) percentage of DCXpositive cells with tertiary dendrites in the SGZ/GCL. $* \mathrm{p}<0.05$ indicates significantly different from wild type control (Student's t-test). 


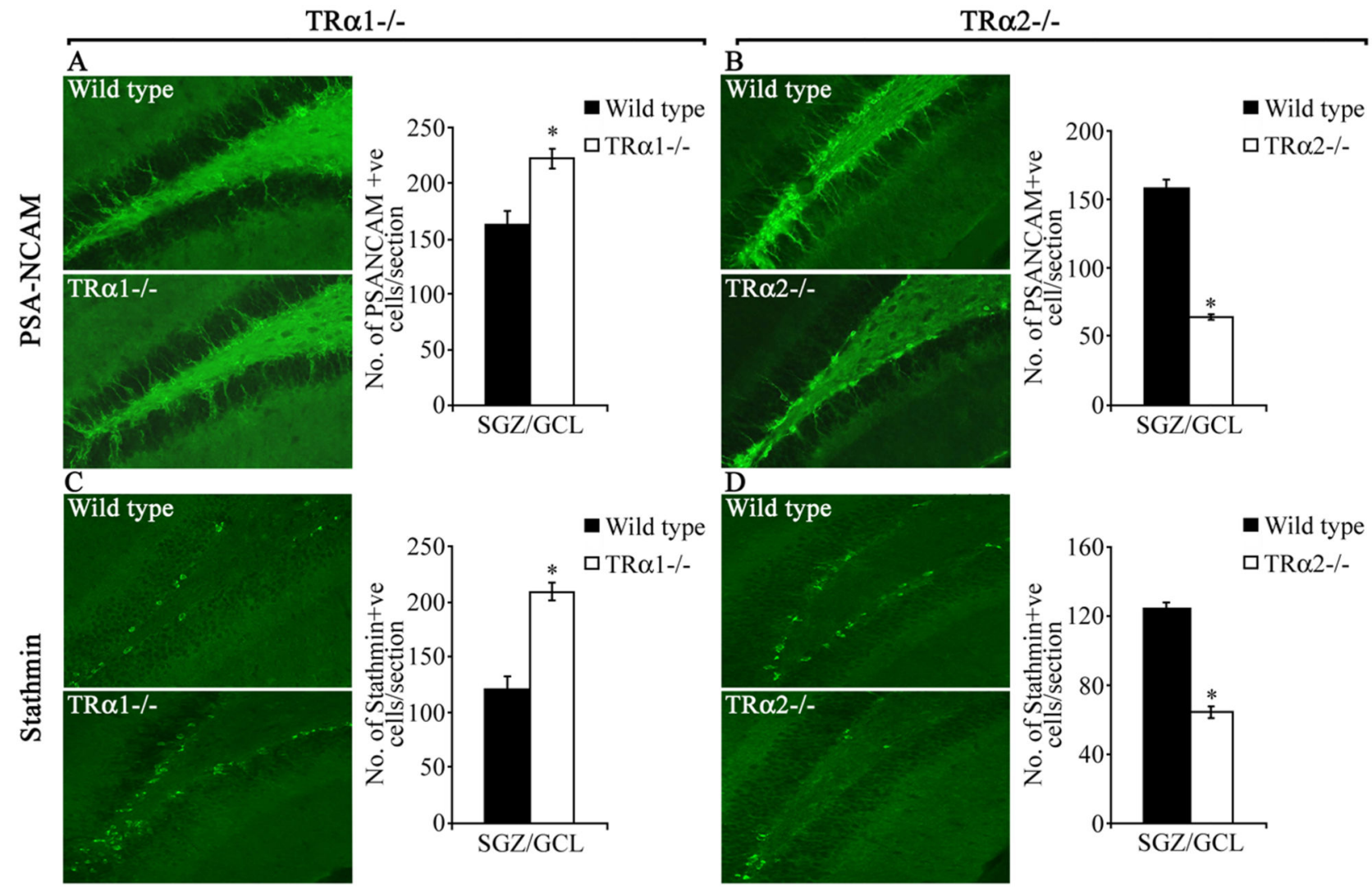

Figure 5.

TRa1-/- and TRa2-/- mice display altered expression of neurogenic niche markers. The number of PSA-NCAM and stathmin-immunopositive cells in TRa1-/- and TRa2-/- mice was assessed using immunofluorescence. Shown are representative immunofluorescence images for PSA-NCAM (A: TRa1-/-, B: TRa2-/-) and stathmin (C: TRa1-/-, D: TRa2-/ -) from TRa1-/- and TRa2-/- mice along with their respective wild type controls at 200x magnification. Quantitative analysis indicated a significant increase in the numbers of both PSA-NCAM and stathmin-positive cells in the subgranular zone (SGZ)/ granular cell layer (GCL) of the TRa1-/- mice as compared to their wild type controls (A- PSA-NCAM, Cstathmin). In contrast, a significant decline in numbers of both PSA-NCAM and stathminpositive cells per section was observed in TRa2-/- mice as compared to their wild type controls (B- PSA-NCAM, D- stathmin). The results are expressed as the mean $\pm \mathrm{SEM}(\mathrm{n}=$ 3-5/group) number of PSA-NCAM- or stathmin-positive cells per section in the SGZ/GCL. $* \mathrm{p}<0.05$ indicates significantly different from wild type control (Student's t-test). 

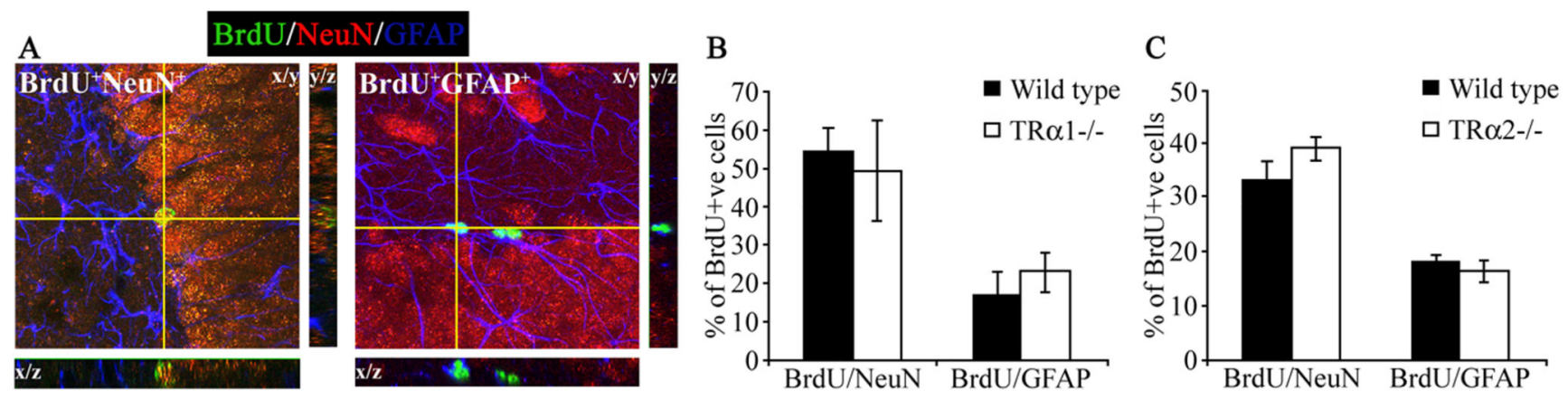

Figure 6.

Differentiation of adult hippocampal progenitors into NeuN-positive neurons or GFAPpositive glia is unaffected in the TRa1-/- and TRa2-/- mice. TRa1-/- and TRa2-/mice, along with their respective wild type littermate controls, received BrdU (150 mg/kg) once daily by intraperitoneal injection for 3 consecutive days and were sacrificed 28 days after the last injection. To examine the neuronal and glial differentiation of newborn adult hippocampal progenitors within the subgranular zone (SGZ) / granule cell layer (GCL) triple immunofluorescence for BrdU, the mature neuronal marker, neuronal nuclei (NeuN), and the glial marker, glial fibrillary acidic protein (GFAP) was performed. Shown (A) are representative merged confocal z-stack images of BrdU colocalization with NeuN (BrdU $+\mathrm{NeuN}+)$ or GFAP (BrdU+GFAP+), with the cell at the crosshairs demonstrating colocalization (600x magnification). Confocal z-stack analysis indicated no change in colocalization of BrdU with either NeuN or GFAP in the TRa1-/- (B) or the TRa2-/- (C) mice as compared to their wild type controls. The results are expressed as the mean \pm SEM ( $n=3-5 /$ group) percent colocalization of BrdU-positive cells with NeuN or GFAP in the SGZ/GCL. 

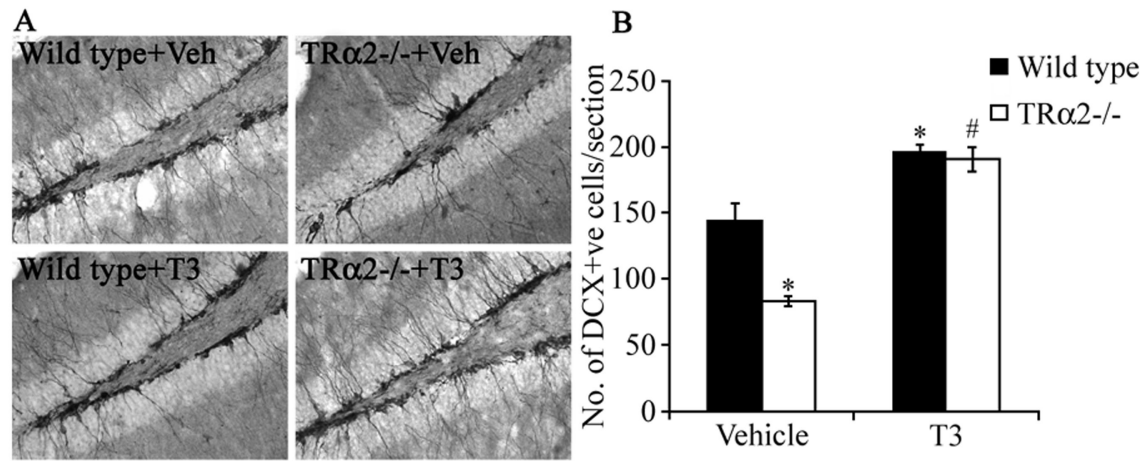

Figure 7.

Thyroid hormone treatment rescues the decrease in the number of doublecortin (DCX)positive immature neurons in the TRa2-/- mice. Wild type and TRa2-/- mice were divided into four experimental groups (Wild type +Veh, Wild type + T3, TRa2-I- + Veh, TRa2-l- + T3). The groups receiving thyroid treatment were given T3 $(0.5 \mu \mathrm{g} / \mathrm{ml})$ in drinking water for 16 days prior to sacrifice. Shown are representative photomicrographs (200x magnification) of DCX immunohistochemistry in the subgranular zone (SGZ) / granular cell layer (GCL) of vehicle and T3-treated wild type and TRa2-/- mice (A). Stereological quantitative analysis revealed a significant decline in the number of cells expressing DCX in the vehicle-treated TRa2-/- mice as compared to the vehicle-treated wild type controls (B). This decrease was rescued by thyroid hormone treatment, as the number of DCX-positive cells in the T3-treated TRa2-/- mice was significantly higher than vehicle-treated TRa2-/- mice (B). There was also a significant increase in the number of DCX-positive cells in the SGZ/GCL of T3-treated wild type mice as compared to vehicle treated wild type controls. The results are expressed as the mean \pm SEM ( $n=4-5 /$ group) number of DCX-positive cells per section in the SGZ/GCL. * $p<0.05$ indicates significantly different from vehicle-treated wild type control group; \#p< 0.05 indicates significantly different from vehicle treated TRa2-/- group (ANOVA and Bonferroni post-hoc test). 


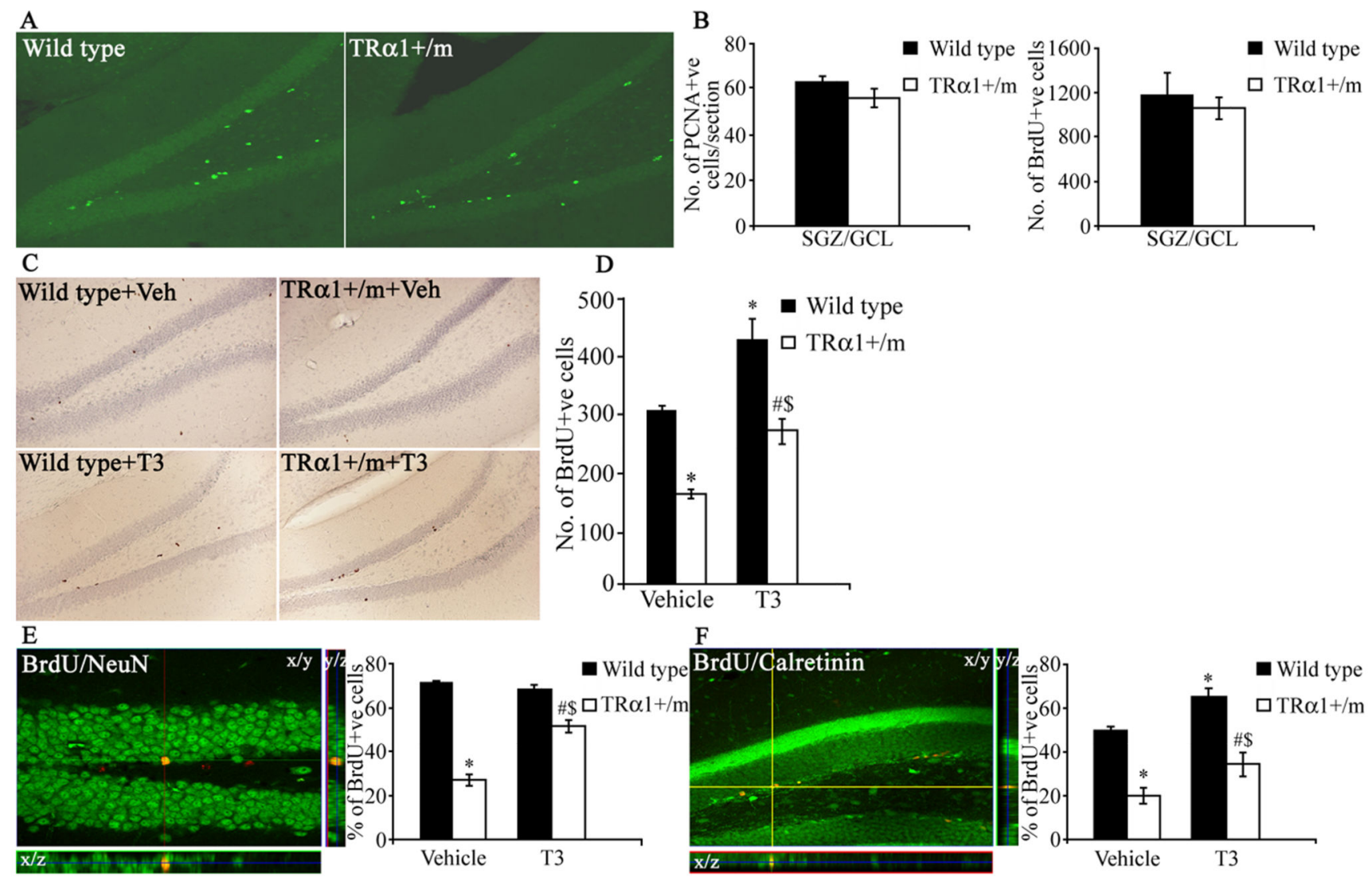

Figure 8.

Postmitotic survival and neuronal differentiation of adult hippocampal progenitors is affected in TRa1+/m (TRa1+/m) mice, and can be rescued by thyroid hormone treatment. In order to assess the proliferation of adult hippocampal progenitors, TRa $1+/ \mathrm{m}$ and wild type littermate controls received a single BrdU $(100 \mathrm{mg} / \mathrm{kg})$ injection and were sacrificed 2 hours later. Adult hippocampal progenitor proliferation was also assessed using immunohistochemistry for the endogenous mitotic marker PCNA. Shown are representative images of PCNA immunofluorescence at 200x magnification from a TRa1+/m mouse and a wild type control (A). PCNA-positive progenitors were observed in clusters within the subgranular zone (SGZ) / granular cell layer (GCL). There was no significant difference in the number of cells expressing PCNA (B) or in the number of proliferating BrdU-positive cells (B) in the SGZ/GCL of TRa $1+/ \mathrm{m}$ mice as compared to their wild type controls. To examine adult hippocampal progenitor survival and differentiation TRa1+/m mice and their wild type controls received daily administration of BrdU $(150 \mathrm{mg} / \mathrm{kg}$ ) for three days and were sacrificed 30 days after the final BrdU administration as described in Materials and Methods. To address whether thyroid hormone treatment could rescue the effects on progenitor survival and differentiation observed in TRa $1+/ \mathrm{m}$ mice, animals were divided into four experimental groups namely: Wild type + Veh, TRa1+/m + Veh, Wild type + T3, $\mathrm{TRa} 1+/ \mathrm{m}+\mathrm{T} 3$. Thyroid hormone treatment was administered in drinking water as described in Materials and Methods. Shown are representative photomicrographs of BrdU immunohistochemistry at 200x magnification in the SGZ/GCL of vehicle and T3-treated 
wild type and TRa $1+/ \mathrm{m}$ mice (C). The number of BrdU-positive cells that persist 30 days after BrdU administration in the SGZ/GCL of TRa1+/m mice was significantly lower than that observed in the vehicle-treated wild type controls (D). T3-treatment restored the decrease in postmitotic survival in the TRa $1+/ \mathrm{m}$ mice, and also significantly increased the number of BrdU-positive cells in the SGZ/GCL of wild type mice compared to vehicletreated wild type controls (D). Neuronal differentiation was assessed by examining the colocalization of BrdU with two markers of neuronal differentiation, NeuN and Calretinin. Shown are representative merged confocal z-stack images of BrdU-NeuN colocalization (E) and BrdU-calretinin colocalization (F) at 400x magnification. Shown at the crosshairs are examples of BrdU-positive cells that colocalize with NeuN (E) and calretinin (F) with orthogonal sections confirming colocalization in the z-plane. The percent colocalization of BrdU with NeuN (E) and with calretinin (F) was significantly reduced in the TRa1+/m mice as compared to wild type controls. Thyroid hormone treatment was capable of partially rescuing the decline in BrdU-NeuN and BrdU-calretinin colocalization observed in $\mathrm{TRa} 1+/ \mathrm{m}$ mice. The results are expressed as the mean \pm SEM ( $\mathrm{n}=4-5 /$ group) number of BrdU-positive cells (B, D), number of PCNA-positive cells per section (B), or percent colocalization of BrdU-positive cells with NeuN (E) or Calretinin (F) in the SGZ/GCL. *p < 0.05 indicates significantly different from vehicle-treated wild type controls; $\# \mathrm{p}<0.05$ indicates significantly different from T3-treated wild type controls; $\$ p<0.05$ indicates significantly different from vehicle treated TRa $1+/ \mathrm{m}$ group (ANOVA and Bonferroni posthoc test). 
A
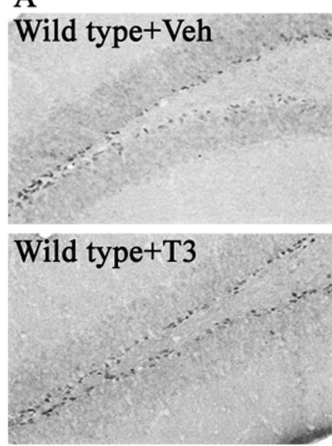

C
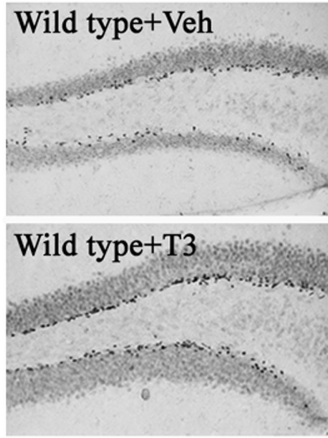
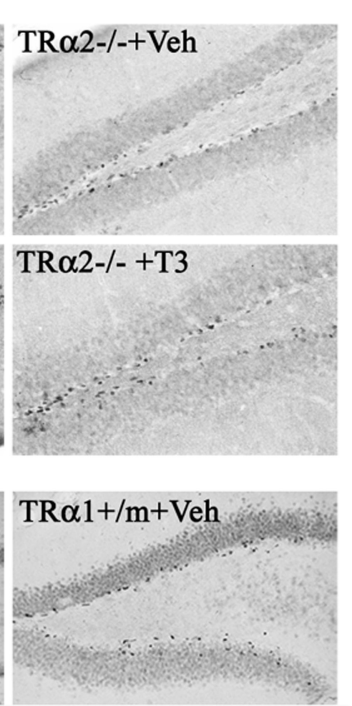

$\mathrm{TR} \alpha 1+/ \mathrm{m}+\mathrm{T} 3$

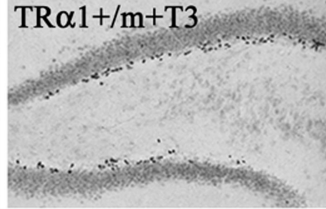

B

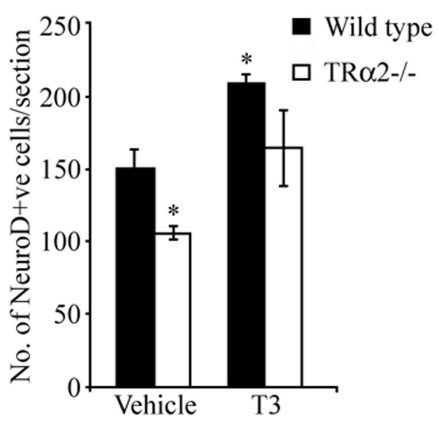

D

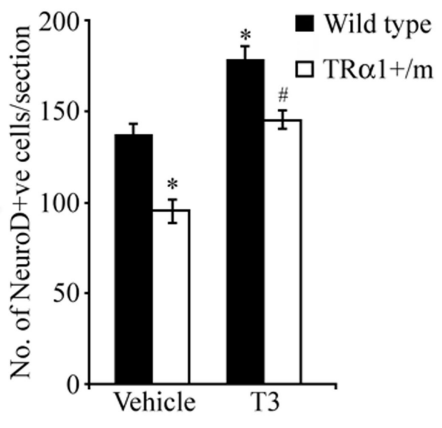

Figure 9.

TRa2-/- and TRa1+/m mice exhibit a significant reduction in the number of NeuroD positive cells within the SGZ/GCL. To assess the effect of thyroid hormone treatment on the number of NeuroD-positive cells in the TRa2-/- and TRa $1+/ \mathrm{m}$ mice, the treatment groups received T3 $(0.5 \mu \mathrm{g} / \mathrm{ml})$ in drinking water as described in Materials and Methods. Shown are representative photomicrographs of NeuroD-expressing cells at 200x magnification in the subgranular zone (SGZ) / granular cell layer (GCL) of vehicle-and T3-treated TRa2-/mice (A) and vehicle- and T3-treated TRa1+/m mice (C) along with their respective wild type controls. There was a significant decrease in the number of NeuroD-positive adult hippocampal progenitors in the SGZ/GCL of the TRa2-/- knockout mice as compared to their wild type controls, which was partially rescued by thyroid hormone treatment but did not reach significance ( $p=0.06$, Bonferroni post-hoc test) (B). Similarly, there was a significant decline in the number of NeuroD-expressing cells in the SGZ/GCL of the vehicle-treated TRa1+/m mice as compared to vehicle-treated wild type controls, which was rescued by thyroid treatment (D). T3-treated wild type mice also showed a significant increase in the number of NeuroD-positive cells compared to vehicle-treated wild type controls (B, D). The results are expressed as the mean \pm SEM ( $n=3-5 /$ group) number of NeuroD-positive cells per section. ${ }^{*} \mathrm{p}<0.05$ indicates significantly different from vehicletreated wild type control; $\# \mathrm{p}<0.05$ indicates significantly different from T3-treated wild type control (ANOVA and Bonferroni post-hoc test). 
A

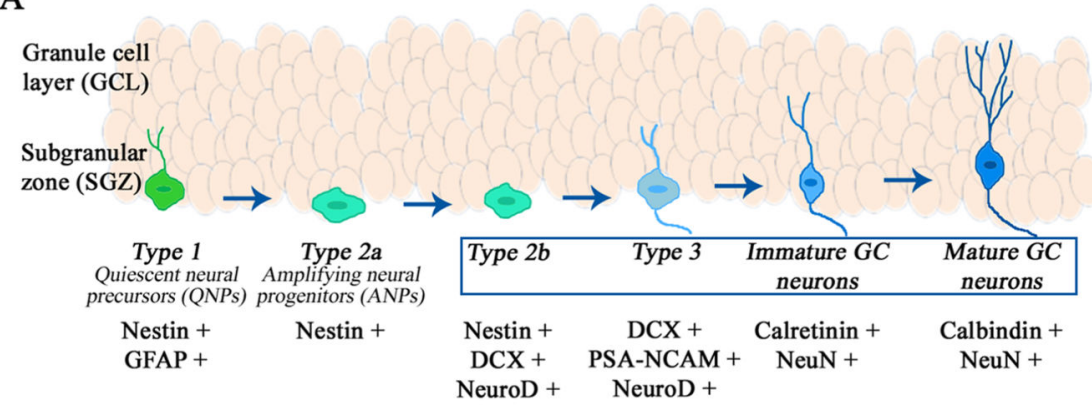

B

$\mathrm{C}$

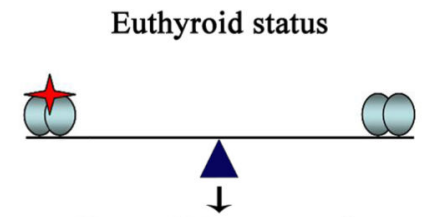

Hypothyroid/TR $\alpha 1$ overexpression/TR $\alpha 1+/ \mathrm{m}$

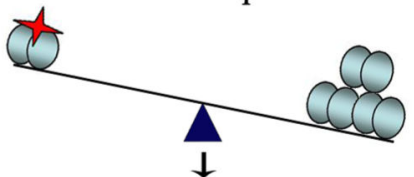

Normal Neurogenesis

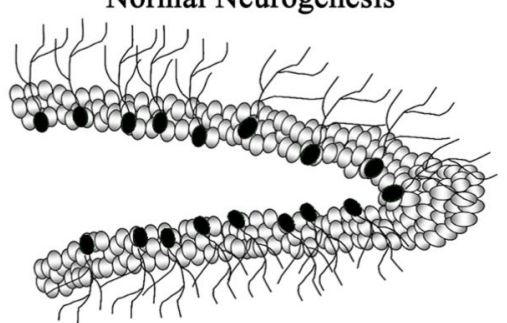

Reduced post-mitotic survival
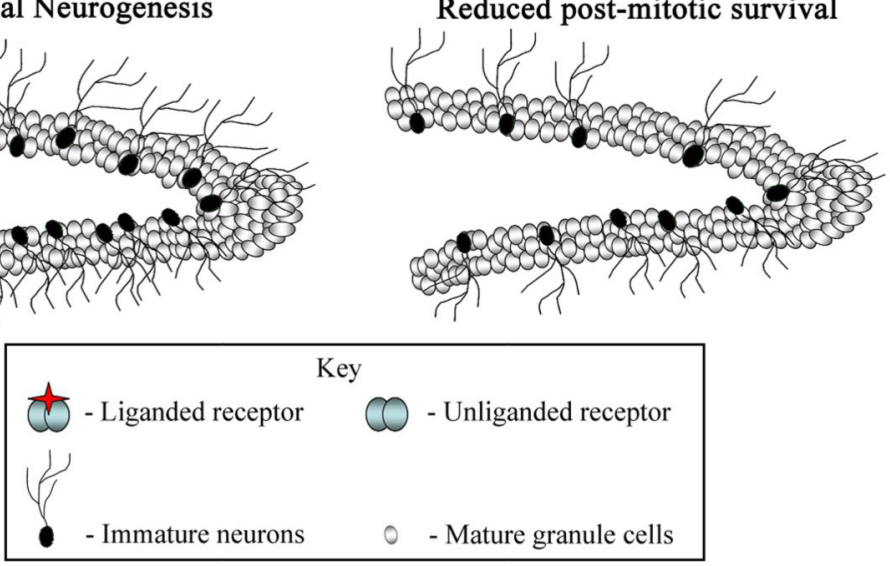

Figure 10.

Shown is a schematic of the developmental stage specific expression of markers by adult hippocampal progenitors (A). Type 1 quiescent neural precursors (QNPs) express Nestin and GFAP, and are the putative stem cells in the adult hippocampus. Type 2a cells, the transiently amplifying neural progenitors (ANPs), express Nestin alone, while Type $2 \mathrm{~b}$ cells express Nestin along with DCX and NeuroD. Type 3 neuroblasts express DCX, PSA-

NCAM and NeuroD, while Immature neurons express Calretinin and NeuN. Mature granule cell neurons express Calbindin and NeuN. The boxed area denotes the stages of hippocampal progenitor development that are sensitive to perturbations of TRa1. (B, C) Schematic of adult hippocampal neurogenesis in an euthyroid animal and in conditions that result in unliganded TRa1 receptor. The schematic shows the dentate gyrus subfield of the hippocampus, containing mature granule cell neurons (white) within the granule cell layer (GCL), the hilus and the neurogenic niche namely the subgranular zone (SGZ) at the border of the hilus and the GCL which contains the adult hippocampal progenitors. Shown are postmitotic neuroblasts (black) in the SGZ that arise from cell division of adult hippocampal progenitors and eventually differentiate into mature granule cells (white) within the GCL of the dentate gyrus. Normal, ongoing hippocampal neurogenesis which occurs in euthyroid 
conditions is schematized in B. Conditions such as hypothyroidism, TRa2-/- mice that overexpress TRa 1 and dominant negative TRa $1(\mathrm{TRa} 1+/ \mathrm{m})$ mutant mice with a 10 fold reduction in affinity for thyroid hormone all result in a decline in adult hippocampal neurogenesis, through a decrease in the survival of postmitotic neuroblasts as illustrated in C. These effects (C) are thought to arise as a consequence of repressor effects on the postmitotic survival of adult hippocampal progenitors induced by a TRa 1 aporeceptor. A change in the ratio of unliganded and liganded TRa 1 receptors may contribute via TRa 1 aporeceptor effects to the impaired adult hippocampal neurogenesis observed in adult-onset hypothyroidism. 